\title{
The Impact on Effectiveness and User Satisfaction of Menu Positioning on Web Pages
}

\author{
Dr Pietro Murano \\ University of Salford, School of Computing, Science and \\ Engineering, \\ Salford, M5 4WT, UK
}

\author{
Kennedy K. Oenga \\ University of Salford, School of Computing, Science and \\ Engineering, \\ Salford, M5 4WT, UK
}

\begin{abstract}
The authors of this paper are conducting research into the usability of menu positioning on web pages. Other researchers have also done work in this area, but the results are not conclusive and therefore more work still needs to be done in this area. The design and results of an empirical experiment, investigating the usability of menu positioning on a supermarket web site, are presented in this paper. As a comparison, the authors tested a left vertical menu and a fisheye menu placed horizontally at the top of a page in a prototype supermarket web site against a real supermarket web site using a horizontal menu placed at the top of a page. Few significant results were observed, which gave rise to the conclusion that overall there were not many differences between the tested menu types. Furthermore, an explanation for the results observed is discussed in terms of cognitive, physical, functional and sensory affordances. It is suggested that observation of the affordances may be a more crucial aspect to menu design than the actual menu positioning.
\end{abstract}

Keywords- Usability; menu design; menu positioning; affordances.

\section{INTRODUCTION}

Most web sites make use of some sort of structure for organising content. The content is then usually accessed by means of various different types of navigation elements. The most common of these tend to be menu-based, where the menu(s) is potentially placed at different locations on the user interface - depending on the designer. Some examples of commonly used navigation schemes are to have menus at the top, bottom, left or right sides of a web page and in some cases combinations of these will also be used. One of the common combinations one can see on certain web sites is to have a horizontal menu at the top of a page and a vertical menu at the left side of the page (inverted-L configuration).

Various dedicated Human Computer Interaction texts also devote some effort in discussing the appropriate design of menus, e.g. Dix, Finlay, Abowd and Beale [4], Benyon [1] and Rogers, Sharp, and Preece [13]. However, despite being able to access guidelines and advice for menu design, the real life situation is that empirical evidence regarding the effectives and user satisfaction of different menu designs is inconclusive overall when various studies are considered as a whole.

While the authors of this paper acknowledge that each type of menu has stylistic aspects to it, we are more concerned with effectiveness and user satisfaction of menu types and their positioning used on web pages. Various studies have been carried out to try and assess the effectiveness and user satisfaction of different menu types. However to our knowledge the results are not completely conclusive and therefore this is still a worthy area of research. We are seeking to contribute to the body of knowledge concerning effectiveness and user satisfaction of menu types and positioning in web pages. Furthermore it is our aim to explain our results in terms of the theory of affordances as expressed by Hartson [7] in the context of user interfaces. Overall this is a very important area of research because the success of a web site involves several different aspects. One of these is the usability of the menus and their positioning. A web site that lacks usability in some form, e.g. having bad menu design and positioning, could lead to loss of business for the owners or simply to not receiving any visitors.

Therefore this paper will firstly discuss some related works in similar areas to our research. This will then be followed by a description of an experiment carried out aiming to determine effectiveness and user satisfaction of different menu positioning on web pages. This will be followed by a presentation of the main results and linked to the theory of affordances [7]. Lastly conclusions and future work will be discussed.

\section{RELATED WORK}

As suggested above, some researchers have been investigating similar issues to the work presented in this paper. However, to our knowledge the overall conclusions regarding effectiveness and user satisfaction are inconclusive and therefore worthy of being studied further.

In a study by Burrell and Sodan [3], menu positioning was investigated using six different menu positions. These were: 1 . A tabbed menu placed horizontally and at the top of the page 2. A horizontal menu placed at the top of the page (not tabbed) 3. A menu placed horizontally at the top of the page and a menu placed vertically at the left side of the page 4 . A vertical menu placed at the left of the page 5. A menu placed horizontally at the top of the page and also a menu placed horizontally at the bottom of the page 6 . A menu placed horizontally at the top of the page and a menu placed vertically at the right side of the page.

The authors then conducted a study with prototype web sites and the above described menu positions. The actual tasks carried out by participants are not clearly indicated in the paper. However it may be that free form exploration was used by the participants so as to experience the different menu 
positions. The authors concluded from their data that the tabbed menu was preferred by participants. While the idea for the investigation of the six menu positions was interesting, this work is lacking in various ways. Some examples include that ideally such a study should also investigate effectiveness by using some carefully designed tasks and measures. This did not seem to be a part of the study. Also, as mentioned above, the details of the tasks are not revealed in the paper and it is therefore difficult to judge if there were any biasing factors in the task design.

In another study by Dos Santos, De Lara, Watanabe, Filho and Fortes [5] eight different types of horizontal menus were tested with participants. The menu types were, with actual names/descriptions:

1) A superfish dropdown menu

2) A mega-dropdown menu

3) A Vimeo style dropdown menu

4) A Simple jQuery Dropdown menu

5) A Sexy dropdown menu

6) 'A different top navigation' menu

7) A horizontal menu 'that creates columns for grouping information in sub-menus' [4]

8) A jQuery $(\mathrm{mb})$ Mепu 2.7.

The study was a within users study (for the menu types) with a between users element (age grouping), i.e. this was a mixed design. The study involved participants carrying out two tasks involving some menu usage. Although the tasks are not clearly described in the paper, the paper indicates that there was an element of 'looking for/finding information' as part of the tasks. Based on these activities, the researchers recorded the average time to complete the tasks, the errors committed by participants and the task completion rate. This data was collected remotely by means of software logging.

The overall results of the work are not entirely clear. The authors' argumentation is mostly centred around averages. However it is not clear in several cases if there are any statistically significant differences observed. A visual inspection of some of the bar charts presented in the paper may indicate some significance, but without the actual data being available it is not possible to categorically confirm this aspect. The authors do argue that their results suggest that menus 1 and 3 (see above) were better. However they do not directly state if this is in terms of fewer errors or faster task completion times, or both. Furthermore the authors' comparisons were restricted to horizontal menus placed at the top of a page, which makes the study limited in nature. Menus placed in other positions on a page should ideally have been investigated.

Another study worthy of consideration is by Leuthold, Schmutz, Bargas-Avila, Tuch, and Opwis [8]. This was a study where the authors compared three types of vertical menu positioned at the left side of the page. These were:

1) A simple menu consisting of clickable links

2) A menu like the simple menu in 1, but with more links which were grouped under various headings ('service navigation items') and
3) A dynamic menu where various headings could be expanded by a user by clicking on a heading, which would reveal further clickable options. This was essentially a compacted version of menu 2.

The authors used eye-tracking equipment to gather data. The context of the web site was a storefront which purported to sell books, DVDs and music. The study involved a series of participants taking part in a simple task and then a more complex task. The tasks basically involved navigating through some of the links to find some information and potential items for purchase. The authors measured user performance, navigational approaches and user preferences.

The authors' results suggested that there was a greater success rate with a first click whilst participants used menu 2 (described above) for the simple and more complex tasks. Also fewer eye fixations were required for the simple and more complex tasks whilst using menu 2. However with menu 1, participants were faster whilst undertaking a simple task and participants were faster with menu 2 during a more complex task. Regarding subjective opinions, overall the authors found that menu 2 was the preferred option.

The research presented by the authors is interesting. However it does have some weaknesses. The first of these is that the menus tested were of the vertical type placed at the left side of the page. It would have been interesting to have tested menus in different positions on the page - although it is accepted that for the purposes of their hypothesis other positions were not necessarily required. Also some of the results are rather obvious in nature, e.g. since menu 3 was a compacted version of menu 2, it is rather obvious that menu 3 would require more clicks to use and therefore more time. Regarding the user satisfaction aspects, we would argue that the measures reported in the paper were rather coarse grained in nature and more detail should have been elicited from the participants in order to reveal detailed perceptions of satisfaction.

A further aspect to consider about the results and the study as a whole is that it gives us some insight into the kinds of links that could be suitable to include in a menu. However this work does not deal with the issue of the actual positioning of menu items and menus on a page.

The last study we will consider in this paper is by Bernard, Hamblin and Chaparro [2]. This was a study where three menu types were evaluated. These were: 1 An index menu, where the menu options appearing as links were all displayed in the centre of the page, 2. A horizontal menu at the top of the page and 3. A vertical menu positioned at the left of the page.

The authors aimed to design realistic tasks based on browsing for information on a web site. Some of the tasks involved finding specific products, while some of the tasks were more vague in nature, because participants were presented with a scenario type context. This context did not specifically give a specific product to find, but some product would be implied in the context given.

The experiment used a between users design and the authors measured task completion time, search efficiency and 
participants' subjective opinions regarding their interaction experience.

Their results showed with statistical significance that the index menu incurred faster times for task completion compared to the other two menus included in the study. Several aspects of participants' perceptions were elicited and most of these were not statistically significant. However participants did indicate that their first choice would be the index menu design. Although it is unclear from [2] if this finding was statistically significant.

Overall the work in [2], in our examination, seems to be one of the more rigorous studies published in this area and is in our opinion the most rigorous we have summarised here. However there were details missing regarding the actual procedure followed during their experiment and it would have been safer to have had a slightly larger participant sample. Also as acknowledged by the authors of [2], the menus used in their study did not descend to very deep levels.

Another aspect of previous work that we wish to briefly summarise concerns the theory of affordances which we will use to explain our observations. The theory of affordances was initially suggested by Gibson [6]. However, over time, some researchers began to apply and extend the theory to user interfaces, e.g. Hartson [7] and Norman [11, 12].

Hartson suggested the existence of cognitive, physical, functional and sensory affordances. He reasoned that when users are doing some computer related task, the users are using cognitive, physical and sensory actions.

Cognitive affordances involve 'a design feature that helps, supports, facilitates, or enables thinking and/or knowing about something' [7]. A simple example of this, concerns presenting feedback to a user that is clear and precise. If a designer labels a button, the label should easily indicate to the user what will happen if the button is clicked.

Physical affordances are 'a design feature that helps, aids, supports, facilitates, or enables physically doing something' [7]. Hartson suggests that a button that can be clicked by a user is a physical object acted on by a human and the button size should be big enough to allow easy clicking. This would therefore be a physical affordance characteristic. Functional affordances concern having some purpose in relation to a physical affordance. A simple example is that clicking on a button should have some purpose with a goal in mind. The opposite is that just clicking anywhere on the screen is not purposeful and has no goal.

Finally, sensory affordances concern 'a design feature that helps, aids, supports, facilitates or enables the user in sensing (e.g. seeing, feeling, hearing) something' [7]. Sensory affordances are linked to cognitive and physical affordances as they complement one another. Therefore the users need to be able to 'sense' the cognitive and physical affordances so that these affordances can help the user.

This brief consideration of some of the key work in this research area (including the theory of affordances), shows that there is still much more work to be done in order to discover more conclusively which menu design may be more effective and satisfying for users. Although other researchers have done some work in this area, often limits in rigour and limitations in the types of menu design tested, show that more evidence needs to be gathered for the benefit of the research community and user interface designers. In the next section we present the details and results of a study where different menu designs were evaluated.

\section{MENU COMPARISON EXPERIMENT}

In order to add to the body of knowledge regarding the usability of different menu types and layouts, a small prototype was developed to simulate a supermarket web site. The prototype used the same colour scheme and products available on the real supermarket web site. This was then compared with the real supermarket web site, with the main varying components being the menu design. The prototype web site used a left vertical menu and a fisheye menu placed horizontally at the top of the page. The real supermarket web site used a horizontal menu placed at the top of the page. Overall the aim was to discover if these differences in menus and their placement on the web page affected user performance and satisfaction.

\section{A. Hypotheses}

We devised several hypotheses around the area of efficiency of use and user satisfaction for the purposes of this experiment. In all cases we were looking for statistically significant differences in the data to be collected.

1) a) $\mathrm{H}_{0}$ : There will be no difference in the number of navigation errors made in using either of the two web sites.

b) $\mathrm{H}_{1}$ : Participants using the prototype supermarket web site will make fewer navigation errors than those using the real supermarket web site.

2) a) $\mathrm{H}_{0}$ : There will be no difference in the ease of use (efficiency) of the two web sites' menu navigation systems.

b) $\mathrm{H}_{1}$ : Participants will find the prototype supermarket web site navigation easier to use (efficient) than the real supermarket web site menu navigation systems.

3) a) $\mathrm{H}_{0}$ : There will be no difference in the participants' satisfaction level between the two web sites.

b) $\mathrm{H}_{1}$ : Participants' satisfaction level for the prototype supermarket web site will be higher than that of the real supermarket web site.

4) a) $\mathrm{H}_{0}$ : There will be no difference between the two web sites for task time.

b) $\mathrm{H}_{1}$ : Participants using the prototype supermarket web site will incur shorter task times.

\section{B. Users}

Since the experiment involved testing aspects of menu design and positioning on a web page, it was deemed important to have participants with a certain amount of experience in using web sites and computers in general. This is because if there happened to be a number of beginners to such activities, these could potentially bias times and outcomes. Therefore: 
- 56 undergraduate students took part in the experiment.

- All participants had not visited the real supermarket web site in the past and had not seen the prototype web site prior to the experiment.

- All participants had basic IT skills.

- All participants had experience with the Internet and online shopping experience.

- All participants were in the 20-40 age range.

These aspects were elicited by means of a carefully designed pre-experiment questionnaire.

\section{Experimental Design}

Since the tasks and 'products' being used within the tasks were the same for both web sites, a between users design was deployed. This would help to avoid the possibility of some 'learning' taking place, regarding the specific products used. Each participant was randomly assigned to one of the two conditions of the experiment. The two conditions were the prototype supermarket web site and the real supermarket web site.

\section{Variables}

The independent variables were (1) the types of menu being investigated (horizontal menu placed at the top of the page, left vertical menu and the horizontal fisheye menu) and (2) the type of task involving using the menus described, in finding a series of typical products sold in supermarkets.

The dependent variables were the participants' performance in carrying out the tasks and their subjective opinions.

The dependent measures were that the performance was measured by examining the time to complete the tasks, the number of errors made and the success level. The success level was determined by observation of the participants' 'behaviour' and interaction with the web sites. This involved making a decision regarding whether a task was completed with ease, completed with difficulty or not completed at all. An error was recorded if a participant deviated from the optimum path to achieve a task by clicking on an incorrect link. This was a good indicator of aspects of the interface that misled the user.

The subjective opinions were measured by means of a post-experiment questionnaire. The time and errors were recorded by using the Morae [10] software suite. The timing was started by clicking the 'record' button when the participant felt ready to begin and the time was stopped when the participant clicked on the home page link of each respective web site. For the context of this study, clicking on incorrect links that did not lead to the expected information, were categorised as errors. Lastly the post-experiment questionnaire that was used for eliciting subjective opinions had a series of sections where the participant responses were made using Likert [9] type scales. The main areas covered by the postexperiment questionnaire were opinions about the navigation styles being tested, ease of learning of the navigation types, ease of remembering one's position on the web pages, efficiency and feelings of satisfaction in using the web sites and their navigation types.

\section{E. Apparatus and Materials}

The experiment took place in a well lit room containing a desk, and three computer chairs.

Two laptops were used in this experiment and for each the screen display was set to a resolution of 1280 by 800 pixels with the colour set to highest (32 bit). Laptop 1 was a Sony Vaio with a 64 bit processor Intel (R) Core TM2 Duo, CPU T6600 $2.20 \mathrm{GHz}$ and $4.00 \mathrm{~GB}$ RAM. This was used by the researcher and was running Morae Observer [10]. The Morae Observer in the Sony laptop connected with Morae Recorder [10] on laptop 2 on a wireless network using an IP address. The two web sites were also run on laptop 2. This was a 32 bit HP Compaq 6735s, with an AMD Sempron, 2.10 GHZ and 2GB RAM. The operating system for both laptops was Windows Vista Home Basic and Internet Explorer 8 was used for the web browser.

Morae Recorder was used for digitally capturing the participants' interaction and Morae Observer was used by the experimenters to observe the participants' interactions in real time without interrupting the participants' interaction in any way.

Five tasks were designed for this experiment. Each centred around typical shopping type activities on a supermarket web site. Further, the information/products participants had to find, involved using the menus to various sub-levels in the hierarchical structure of the web sites. To introduce the tasks and make them more realistic a small scenario was presented to the participants, as follows:

You intend to buy a few Christmas presents for your younger brother. These items are to be purchased online as the shops are overcrowded around this time when people are busy with their Christmas shopping. The Items you require are available at the supermarket's online shop. You are interested in buying a packet of milk, an iPod and a Scooter. You are not required to purchase the items and for this experiment only, you do not use the search button to get to the products.

Therefore the five tasks were as follows:

Task 1: Use the navigation links to locate Whole Milk $1.13 L$ (2 pint) and add it to the shopping cart. Then click on the home button to end this task.

Task 2: Use the navigation links to locate the New iPod Shuffle 2GB - Pink and add it to the shopping cart. Then click on the home button to end this task.

Task 3: Start the task from the Horizontal link buttons, and then the Icons that appear in the subsequent pages (do not use the left menu buttons) to locate Lightning Strike Scooter Pink and add it to the shopping cart. Then click on the home button to end this task.

Task 4: This task is a continuation from tasks 1,2 and 3 above. Each time you purchase an item from the supermarket, you can collect loyalty points. How can you get double loyalty points from your purchase? The researcher will provide you with paper to write your answer. 
Task 5: What is the difference in Giga Bytes (GB) between the new iPod Shuffle and New iPod Nano Silver (pictures provided). Write your answer on the paper provided by the researcher.

\section{F. Procedure}

The procedure followed and described in this section was initially pilot tested with three independent individuals. The pilot testing showed that the designed procedure was accomplishing the objectives of the study without any obvious problems.

Therefore, each participant was asked to present themselves to a specific room in the institution set aside for the experiment. During the experiment each participant was seated at the desk in the room with the laptop facing them and the researcher sat opposite the participant with the second monitoring laptop facing the researcher.

Each participant was briefed about the web applications and it was stated that the study was evaluating the web applications rather than the participants. Participants were told that the tasks started from the home page and that after adding the items to the shopping basket, each task ended when the participant pressed the home button on the website. Then the participants were given instructions on how to perform the tasks.

They were also asked to complete an informed consent form and fill out a pre-experiment questionnaire that included questions about demographics and computer skills.

A piece of paper was provided for the participants to write answers for tasks four and five. The researcher ensured that the participants started from the home page and ended their tasks by pressing the home button.

The researcher explained that the amount of time taken to complete each task would be measured and that they should not engage themselves in any exploratory behaviour outside the task flow until after the experiment had been completed. Participants were also given the opportunity to ask questions.

Then the participants were given the tasks to do in the order described in the previous section. Time was allowed for them to read the tasks and understand them fully before they started. There were five tasks for each web site and the participants were free to follow any navigation route they wished, except for task three which required them to use the horizontal fisheye menu first (see Variables section above for a description of the dependent measures and how they were recorded).

After completing the tasks, the participants were prompted to fill out an electronic post-experiment questionnaire concerning user satisfaction (see Variables section above for a summary of the areas covered by the post-experiment questionnaire).

\section{G. Results}

In this section, for brevity, only the significant results are presented. For the data collected, the distributions were examined which included the respective means (M) and standard deviations (SD). Then the data was subjected to
Multifactorial Analysis of Variance (MANOVA) testing and where significance was found, the significance was confirmed by means of post-hoc testing using either t-tests or Tukey HSD tests (post-hoc tests not included in this paper for brevity).

For task 4, which involved aspects of finding double loyalty points, there was a significant difference for the number of errors committed, where the prototype supermarket web site $(\mathrm{M}=0.93$ errors, $\mathrm{SD}=0.77)$ incurred significantly $(\mathrm{F}$ $(5,50)=3.26, \mathrm{P}<0.05)$ more errors than the real supermarket web site $(\mathrm{M}=0.39$ errors, $\mathrm{SD}=0.63)$.

The subjective question concerning overall ease of navigation was scored by participants using a Likert [9] type scale of $1-5$, where 5 was the most positive response possible and 1 was the most negative response possible. This question incurred a significant difference in opinions between the various age groups which took part in the experiment. The slightly older groups in both experimental conditions $(\mathrm{M}=$ 3.75 for 36-45 age group, $\mathrm{M}=3.36$ for 31-35 age group) rated the ease of navigation significantly $(\mathrm{F}(5,50)=2.60, \mathrm{P}<0.05)$ less easy than the younger groups in both experimental conditions ( $\mathrm{M}=4.08$ for $25-30$ age group, $\mathrm{M}=4.61$ for $19-24$ age group).

The subjective question concerning the web sites being easy to learn to use by anyone was scored by participants using a Likert [9] type scale of 1-5, where 5 was the most positive response possible and 1 was the most negative response possible. This question incurred a significant difference in opinions across the two web sites. The prototype supermarket web site $(\mathrm{M}=3.39$ opinion score, $\mathrm{SD}=1.31)$ incurred significantly $(\mathrm{F}(5,50)=2.71, \mathrm{P}<0.05)$ lower/more negative opinion scores than the real supermarket web site (M $=4.32$ opinion score, $\mathrm{SD}=1.12$ ).

The subjective question concerning the web sites' navigation being suitable for all levels of users was scored by participants using a Likert [9] type scale of 1-5, where 5 was the most positive response possible and 1 was the most negative response possible. This question incurred a significant difference in opinions across the two web sites. The prototype supermarket web site $(\mathrm{M}=2.89$ opinion score, $\mathrm{SD}=$ $1.59)$ incurred significantly $(\mathrm{F}(5,50)=3.89, \mathrm{P}<0.01)$ lower/more negative opinion scores than the real supermarket web site $(\mathrm{M}=3.68$ opinion score, $\mathrm{SD}=1.28)$.

The subjective question concerning the text size used for menu labelling and the ease of reading such labels, was scored by participants using a Likert [9] type scale of $1-5$, where 5 was the most positive response possible and 1 was the most negative response possible. This question incurred a significant difference $(\mathrm{F}(5,50)=2.76, \mathrm{P}<0.05)$ in opinions between the various age groups which took part in the experiment - across the two experimental conditions $(\mathrm{M}=1.25$ for 36-45 age group, $\mathrm{M}=3.21$ for 31-35 age group, $\mathrm{M}=2.75$ for 25-30 age group and $M=2.44$ for 19-24 age group).

The subjective question concerning a lack of willingness to use the web sites in the future was scored by participants using a Likert [9] type scale of 1-5, where 5 indicated full agreement in not wanting to use the web sites again and 1 indicated complete disagreement in that participants would want to use 
the web sites again. This question incurred a significant difference in opinions across the two web sites. The prototype supermarket web site $(\mathrm{M}=3.32$ opinion score, $\mathrm{SD}=1.19)$ incurred significantly $(\mathrm{F}(5,50)=3.32, \mathrm{P}<0.05)$ more positive opinions towards being willing to use the web site in the future than the real supermarket web site $(\mathrm{M}=4.25$ opinion score, $\mathrm{SD}=1.08)$.

The next section will discuss the results presented above in relation to the hypotheses already presented above and the theory of affordances [7].

\section{H. Experiment Results Discussion}

Overall the significant results presented above, if taken in isolation from the other analysed 'variables' in the experiment, suggest the original real supermarket web site was preferred over the prototype supermarket web site. However the authors feel that the context of the many other subjective questions (see the Variables section above for a summary of the areas covered by the post-experiment questionnaire) asked should not be ignored. These other questions gave insignificant results across the two conditions being tested. This clearly suggests that overall opinion across the two conditions was rather uniform in nature with only a minimal amount of factors showing some statistical significance.

Furthermore, one of the significant results suggested a preference for the prototype supermarket web site in the context of being willing to use the web site in the future. It seems that the prototype supermarket web site in some way fostered some positive emotion in users as they indicated a stronger feeling of wanting to come back to the site.

Therefore due to the results not being so clear cut in terms of all the 'variables' under analysis, we cautiously accept Hypothesis 3(a) $-\mathrm{H}_{0}$, which stated that there would be no difference in the participants' satisfaction level between the two web sites.

In addition, as can be seen in the previous section, significantly more errors were incurred with the prototype supermarket web site. This was specifically for Task 4. However, there were 5 tasks overall and clearly the other 4 tasks did not show any significant differences for errors. Also across the 5 tasks, the times taken to complete tasks did not show any significant differences across the different types of menus/the two web sites. Lastly, for each task, the success levels were also recorded and these did not show any significant differences across the two experimental conditions being tested.

Therefore, due to the results also not being so clear cut regarding the performance in the tasks (with the exception of the errors in Task 4), we cautiously accept Hypotheses 1(a) $\mathrm{H}_{0}$ and 4(a) $-\mathrm{H}_{0}$. These stated that there would be no difference in the number of navigation errors made in using either of the two web sites and that there would be no difference between the two web sites for task time.

We also accept Hypothesis 2(a) - $\mathrm{H}_{0}$ which stated that there would be no difference in the ease of use (efficiency) of the two web sites' menu navigation systems. The authors feel that overall there were not enough significant results in terms of the participant subjective responses and aspects of task time and success level in the tasks.

Lastly, as described in the previous section, we did observe some significant differences within the age groups of the participant groups. While this is not 'age related' research, we could not find any particular explanation for these findings, as the recruitment process did attempt to recruit participants with similar IT skills. However this could be a worthy avenue of further research for 'age related' studies.

Having linked back to the initial hypotheses (see Hypotheses section above), we are also interested in understanding the reasons for few significant results and therefore mostly no large differences between the different menu types. While there could be issues in the experimental design and execution, retrospective examination of the experimental design and execution reveal no obvious confounding variables. However, an examination of the menu types in relation to the theory of affordances as rendered by Hartson [7] could reveal some light on the matter.

Task 4 was the only task out of five tasks to have significant differences in terms of errors, even though for both web sites the information was only two clicks away from the starting point of the task. We suggest that the menu options needing to be chosen to reach the 'answer', perhaps violated the cognitive affordances, by not being labelled with a term that could indicate that double points information was available by clicking a certain menu option. Also the other tasks involved selection of menu options, for both web sites, that had labels which more clearly indicated the path to be followed and therefore observed the cognitive affordances more appropriately.

Regarding the participants' perceptions of the user interfaces used in the experiment, as discussed above, few significant results were observed. We would suggest that this is because the cognitive affordances were mostly equivalent for both web sites, e.g. in most cases the labelling of menu options was relatively clear for supermarket web sites of this kind. This therefore resulted in the 'side effect' of very few significant differences for user satisfaction. Also, we would suggest that the physical affordances were approximately equivalent for both web sites, because the sizing of the menus and each menu option, were of a size that made it easy to select an option. Finally we would suggest that the functional and sensory affordances were also largely equivalent for both web sites. The menu options had clear 'purpose' and worked as intended. Also as discussed above, the designs of both web sites had good visual clarity.

\section{CONCLUSIONS AND FUtURE WORK}

The results suggest overall that particularly in a supermarket shopping web site context, whether the menu is placed horizontally at the top of the page or is replaced with a horizontal fisheye menu and a left vertical menu does not seriously affect interaction time, accuracy and subjective perceptions. Furthermore, we would suggest that irrespective of menu positioning, designers should ensure as far is possible that the cognitive, physical, functional and sensory affordances are not violated in any way, as any violation of 
these could be more crucial than the actual positioning of the menu itself. However the authors of this paper suggest that more work still needs to be done to obtain empirical results for other types of menus and also to investigate in more depth issues of sub-menus and nesting. We suggest this because clearly other researchers have had different results which may indicate that there are other issues at play still to be discovered.

Also future experiments with more difficult tasks could lead to more understanding of the issues. This approach may show clearer results favouring a particular type of menu design. Furthermore more investigation needs to be done in relation to the theory of affordances [7] to gain more evidence that violation of the affordances creates more problems than the actual menu positioning. Lastly we suggest that perhaps other psychological aspects and/or user experience could have an effect that we have not identified yet.

\section{REFERENCES}

[1] D. Benyon, Designing Interactive Systems A Comprehensive Guide to $\mathrm{HCI}$ and Interaction Design, $2^{\text {nd }}$ Edition, Addison Wesley, 2010.

[2] M. L. Bernard, C.J. Hamblin and B.S. Chaparro, Comparing Cascading and Indexed Menu Designs for Differences in Performance and Preference, Proceedings of the Human Factors and Ergonomics Society $47^{\text {th }}$ Annual Meeting -2003 .

[3] A. Burrell and A.C. Sodan, Web Interface Navigation Design: Which Style of Navigation-Link Menus Do Users Prefer?, Proceedings of the $22^{\text {nd }}$ International Conference on Data Engineering Workshops, ICDEW 2006, 3-7 April 2006, Atlanta, GA, USA, IEEE Computer Society.

[4] Dix, A, Finlay, J, Abowd, G.D and Beale, R, Human Computer Interaction, Pearson/Prentice Hall, 2004.
[5] E.P.B. Dos Santos, S.M.A. De Lara, W.M. Watanabe, M.C.A. Filho and R.P.M. Fortes, Usability Evaluation of Horizontal Navigation Bar With Drop-Down Menus by Middle Aged Adults, 29th ACM International Conference on Design of Communication, SIGDOC 2011, October 3-5, Pisa, Italy.

[6] J.J. Gibson, The Ecological Approach to Visual Perception, Houghton Mifflin Co. 1979.

[7] H.R. Hartson, Cognitive, Physical, Sensory and Functional Affordances in Interaction Design, Behaviour and Information Technology, Sept-Oct 2003, 22 (5), p.315-338.

[8] S. Leuthold, P. Schmutz, J.A. Bargas-Avila, A.N. Tuch, and K. Opwis, Vertical Versus Dynamic Menus on the World Wide Web: Eye Tracking Study Measuring the Influence of Menu Design and Task Complexity on User Performance and Subjective Preference, Computers in Human Behaviour, 27(2011) 459-472.

[9] R.A. Likert, Technique for the Measurement of Attitudes, Columbia University Press, NY, 1932.

[10] Morae, http://www.techsmith.com/morae.html, Accessed Feb 2012.

[11] D.A. Norman, Affordance, Conventions, and Design, Interactions, MayJune, 1999, p.39-42.

[12] D.A. Norman, The design of Everyday Things, Basic Books, 2002.

[13] Y. Rogers, H. Sharp, and J. Preece, Interaction Design Beyond Human Computer Interaction, $3^{\text {rd }}$ Edition, Wiley, 2011.

\section{AUTHORS PROFILE}

Dr Pietro Murano is a Computer Scientist at the University of Salford, UK. Amongst other academic and professional qualifications he holds a PhD in Computer Science. His specific research areas are in Human Computer Interaction and Usability of software systems.

Kennedy K. Oenga is a Computer Scientist. He has obtained an MSc in Databases and Web-Based Systems from the University of Salford, UK. One of his research interests is in Human Computer Interaction. 